\title{
Investigation of the ultrasonic sound signals during the cementing materials induration
}

\author{
Vladimir Gaponov ${ }^{1}$, Dmitry Kuznetsov ${ }^{1}$, Vitaly Dudnik ${ }^{1}$, and Nadiia Afanasieva ${ }^{1{ }^{*}}$ \\ ${ }^{1}$ Don State Technical University, Department of Industrial Safety, 344000 Rostov-on-Don, Russian \\ Federation
}

\begin{abstract}
The paper is devoted to investigation mechanism of cement mixture setting and stiffening. Method for registration of the acoustic oscillations induced during the heterophase transitions executing in liquid medium was proposed (method of acoustic emission (AE)). Using of conical vessel serving as a resonator allows the amplification of registered acoustic signal and provides high sensitivity of the registration. The experimental results show that the using of AE-method allows remote monitoring of the dynamics of concrete mixtures condition during setting. Therefore, this method can be successfully applied in building as a nondestructive method for determining the start and end of the solidification of the cement solution.
\end{abstract}

\section{Introduction}

There is a set of complex physical and chemical processes occur during the cementing substances interaction, especially during the cement clinker and water interaction. Mechanism of the cementing substances setting and stiffening is based on exothermal processes of interaction with water. As a result the water-cement paste transform into a dense stony material [1-2]. Method for registration of the acoustic oscillations induced during the heterophase transitions holding in liquid medium was proposed for the considered processes investigation (method of acoustic emission (AE)). Various authors [25] pay attention to the efficiency of AE-method coupled with optical method and computer simulation of crystals forming during the gypsum $\mathrm{CaSO}_{4} \cdot 0,5 \mathrm{H}_{2} \mathrm{O}$ and water interaction.

The cement hardening has a complex mechanism. Chemical reactions between the cement components start immediately after the cement mix and water are blending. The cement particles begin to dissolve and simultaneously the hydration and hydrolysis of resolving products starts. The known research methods mostly used for the considered physical and chemical processes such as static method of the structural and mechanical properties investigation, roentgen phase analysis, local microanalysis etc. provide only integral data and do not provide any time-dependent information. Compare to these methods the AE-method provides information on the cement mixture condition every second. The extended theoretical and experimental analysis of the acoustic oscillations

* Corresponding author: nadezda.a.afanasyeva@gmail.com 
registration methods during the heterophase transitions holding in liquid medium presented in $[1-3]$.

\section{Methodology}

In the present paper, the processes of mixing the building mixture based on cement were studied using the AE-method coupled with heat monitoring. AE-method is a passive method of monitoring. The ultrasonic oscillations provide information on microscopic motions of elastic waves produced by an abrupt deformation of stressed material. Thus the AE-method allows to record timing dynamics of processes that take place in building mixture during its mixing. Additionally, the heat monitoring allows to obtain a thermogram of the building mixture mixing.

As an experimental was considered a concrete mix with grade of 400 and with next proportion of cement, sand and macadam 1:1,6:3,2. The acoustic emission monitoring was conducted by using the A-line $32 \mathrm{D}$ electronic device. The thermogram was obtained by using infrared imager Sat Hotfind D. Compare to the standard methodology of AE-signals registration the methodology presented in current paper supposes to use a conical vessel that serves as a resonator and provide amplification of registered acoustic signal. Capacity was installed on piezosensor that works at frequency range $30-500 \mathrm{kHz}$. The vessel was made by material with low factor of sound attenuation (quartz). The following AEparameters was estimated within the study (Figure 1):

Amplitude of emission - the maximum magnitude of emission signal during the given time interval.

The electrical signal duration $\mathrm{T}_{0}[\mathrm{~s}]$ - the time during which the AE electrical impulse envelope exceeds limiting threshold. The signal time variation range: $10^{-4} \ldots 10^{-8} \mathrm{~s}$.

Rise time $\mathrm{DT}_{\mathrm{r}}[\mathrm{s}]$ - time interval betwing the moment when AE-impulse envelope appears over limiting threshold and the moment when it achieves its maximum amplitude.

Total count $\mathrm{N}_{\Sigma}$ [pulse] - the number of recorded AE-impulse exceeding the discrimination level (limiting threshold).

Infrared imager Sat Hotfind D Central data-processing station

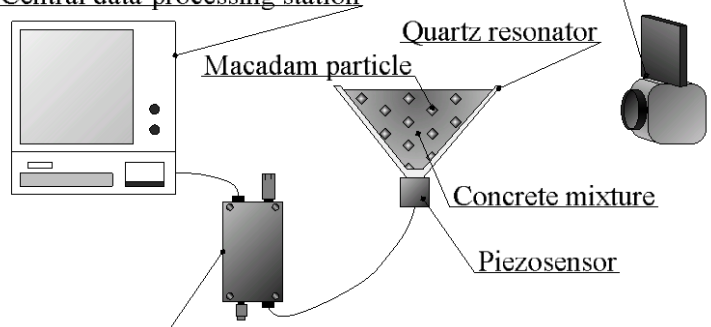

The AE-data collection and processing module

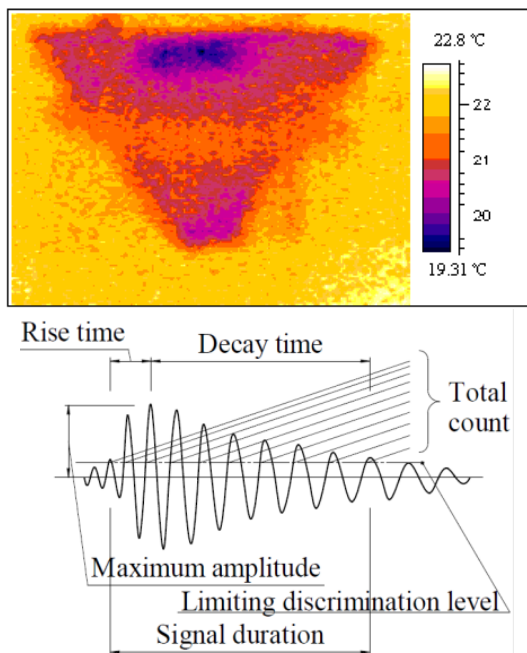

Fig. 1. Schematic of the AE-parameters measuring during the concrete mixture mixing (Source: Author's own figure).

Count rate $\mathrm{N}$ [pulse/s] - time derivative of the AE total count. Variation range: $0 \ldots 10^{15}$ [pulse/s]. 
Electric signal energy Ec $[\mathrm{J}]$ - the measured area under the AE electrical signal envelope.

\section{Results}

The obtained results showed that infrared imager Sat Hotfinds do not recorded any temperature decrease. The observation is caused by the heat exchange with environment and by relatively low exothermicity of the reactions. Therefore only acoustic parameters were studying. The plot presented on figure 2 shows the change in integrated index - total count $\mathrm{N}_{\Sigma}$ within 16 hours from the moment of concrete mixture mixing at the temperature of $30{ }^{\circ} \mathrm{C}$. Another integrated index presented here is a compressive strength of concrete sample.

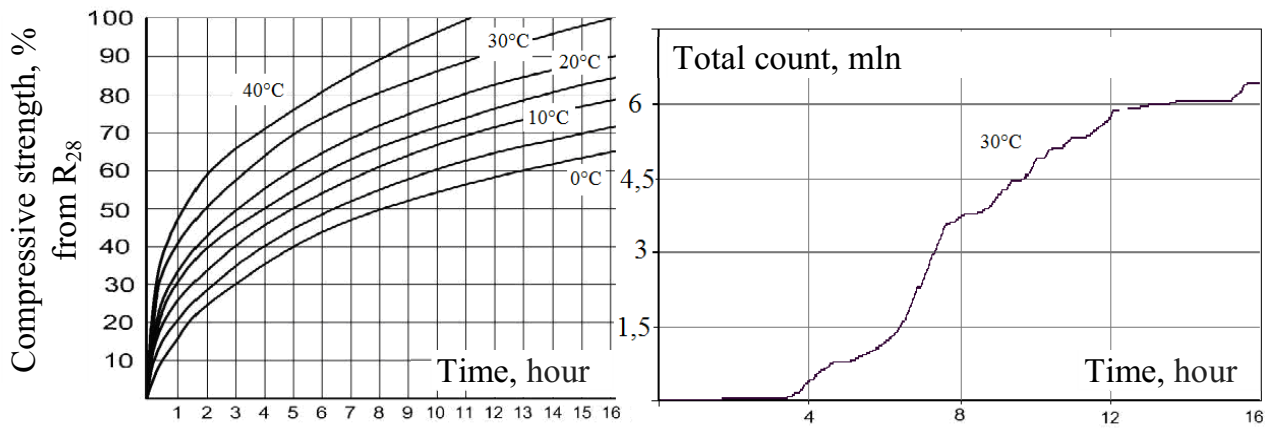

Fig. 2. Change in total count and compressive strength within 16 hours from the moment of concrete mixture mixing [1].

The first period ( $0-3$ hours) can be called a period of dissolution or a preparatory period.

When the cement interacts with water, a chemical reaction occurs on the surface of the granules. The observed monotectic transformation is accompanied by the emission of acoustic signals in the ultrasonic frequency range. Reaction products pass into the solution until the liquid surrounding the cement granules becomes a saturated solution of the reaction products. During this period, the parameters of the AE change insignificantly, the growth of AE activity is observed only after the 3rd hour. Then begins setting, which ends after 5-10 hours after mixing. During the second period (colloidation), the water-cement paste thickens, loses mobility, but strength is not great yet. During the experiments, it was found that approximately 3 hours after the start of the second stage, it can be saying that the concrete/cement was stiffened. Acoustic data indicate that in the period after the 3rd hour from the moment of mixing, a pronounced peak of $\mathrm{AE}$ activity is observed. This process executes a little more than 60 minutes. In addition, this process is clearly visible on the plot of change in AE pulses energy (see Fig. 3). At present, the water-cement paste setting is a well-studied process [5]. 


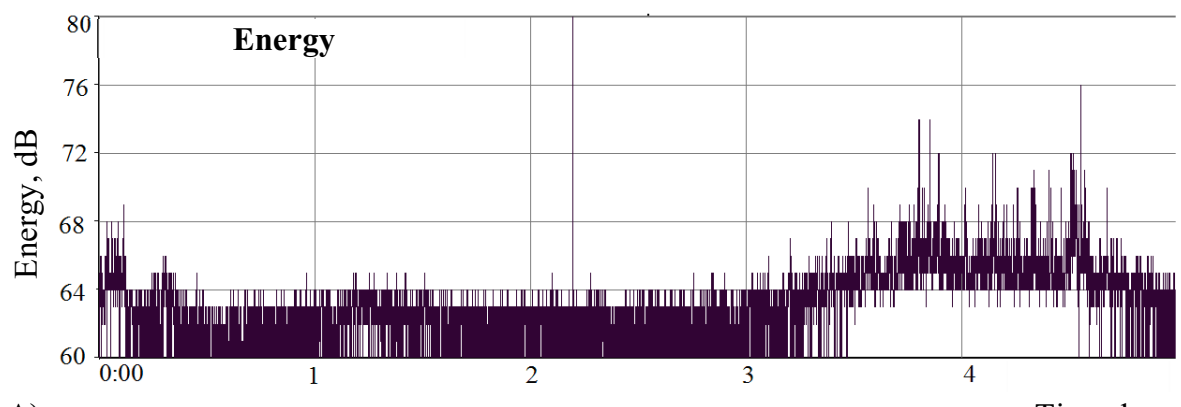

A)

Time, hour

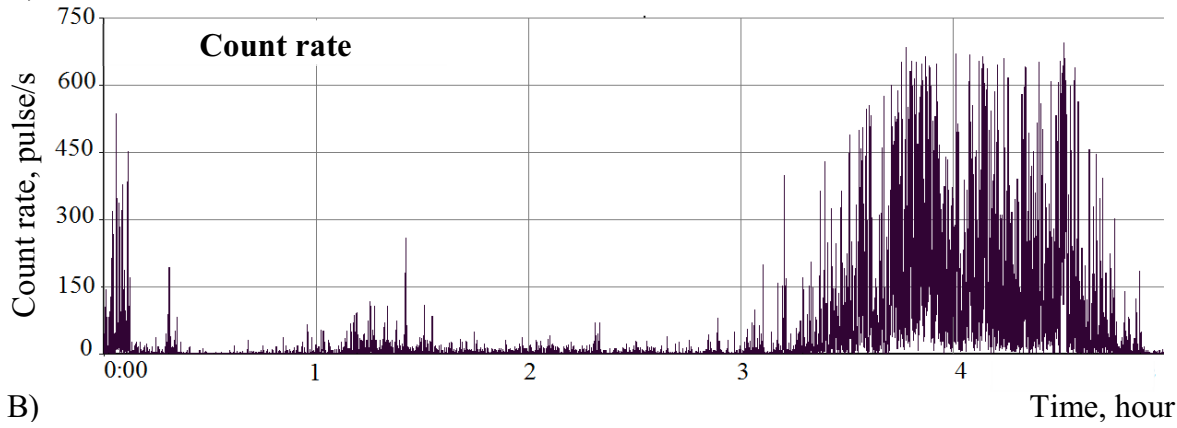

Fig. 3. Change in the $\mathrm{AE}$ parameters during the initial time period (0-5 hours) after the concrete mixture mixing. $\mathrm{A}$ - the change in $\mathrm{AE}$ pulses energy $\mathrm{E}_{\mathrm{c}} ; \mathrm{B}$ - the change in $\mathrm{AE}$ count rate $\mathrm{N}$ (Source: Author's own figure).

It should be noted that in process of further hydration, the amount of free water in the water-cement paste decreases, the stickiness of the gel increases. The cement paste is thickening and setting. Then the new formations start to crystallize. AE-method allows observing the peritectic transformation i.e. the process of solid phase formation as a result of interaction between the liquid phase and initial solid phase, which differs from the new one by its composition and structure. This peritectic transformation can be characterized as follows. The crystals formed are splicing together, overgrowing with long needle crystals and finally a crystalline splice is formed, i.e., the setting of cement becomes finished. Hereafter, the cement stone is compacted due to the ongoing reactions of interaction between cement and water, partial dehydration and further crystallization. A detailed description of the process is available in [1-3].

The main ongoing chemical reactions can be described as follows. Tricalcium silicate $3 \mathrm{CaO} * \mathrm{SiO}_{2}$ (abbreviated as $\mathrm{C}_{3} \mathrm{~S}$ ), interacting with water, forms hydrosilicate and calcium hydroxide $2\left(3 \mathrm{CaO} * \mathrm{SiO}_{2}\right)+6 \mathrm{H}_{2} \mathrm{O}=3 \mathrm{CaO}^{2} 2 \mathrm{SiO}_{2} * 3 \mathrm{H}_{2} \mathrm{O}+3 \mathrm{Ca}(\mathrm{OH})_{2}$. Then the dicalcium silicate is hydrated $2 \mathrm{CaO}^{*} \mathrm{SiO}_{2} \quad\left(\mathrm{C}_{2} \mathrm{~S}\right)$ : $2\left(2 \mathrm{CaO} * \mathrm{SiO}_{2}\right)+4 \mathrm{H}_{2} \mathrm{O}=3 \mathrm{CaO} * 2 \mathrm{SiO}_{2} * 3 \mathrm{H}_{2} \mathrm{O}+\mathrm{Ca}(\mathrm{OH})_{2}$. The tricalcium aluminate, interacting whith water, forms a calcium hydroaluminate: $3 \mathrm{CaO}^{*} \mathrm{Al}_{2} \mathrm{O}_{3}+6 \mathrm{H}_{2} \mathrm{O}=3 \mathrm{CaO} * \mathrm{Al}_{2} \mathrm{O}_{3} * 6 \mathrm{H}_{2} \mathrm{O}$. Four-calcium alumoferrite, interacting with water, break up into hydroaluminate and hydroferrite: $\quad 4 \mathrm{CaO}^{*} \mathrm{Al}_{2} \mathrm{O}_{3} * \mathrm{Fe}_{2} \mathrm{O}_{3}+\mathrm{mH}_{2} \mathrm{O}=3 \mathrm{CaO}^{*} \mathrm{Al}_{2} \mathrm{O}_{3} * 6 \mathrm{H}_{2} \mathrm{O}+\mathrm{CaO} * \mathrm{Fe}_{2} \mathrm{O}_{3} * \mathrm{nH}_{2} \mathrm{O}$. The hydroaluminate is bonding by the addition of natural gypsum, and the hydroferrite becomes a part of the cement gel. The registered changes in AE-parameters for time intervals from 5 to 10 hours from the moment of cement mixing are presented in the Fig. 4. The data obtained indicate that the described process has a large number of intermediate stages. The observation was expectable due to molecular nature of chemical reactions that take place. Since reactions with a molecular structure of more than three are impossible, the 
registration of AE-parameters makes it possible to differentiate the complex chemical process in time.
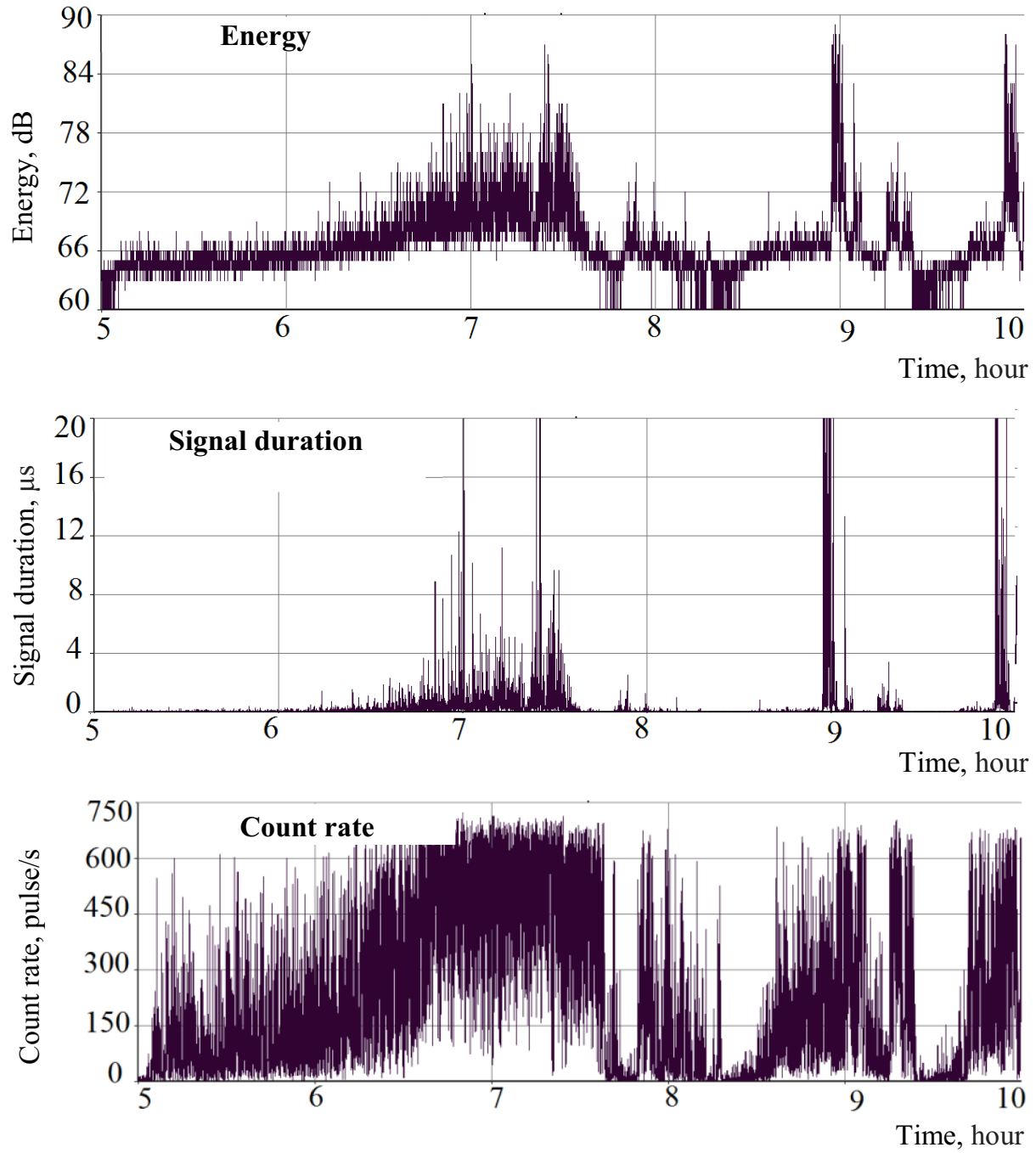

Fig. 4. Change in AE-parameters during setting and hardening of concrete mixture during the time intervals from 5 to 10 hours after mixing.

Further observations on the hardening concrete mixture conditions are reflected at Fig. 5. The high sensitivity of the AE-method made it possible to record the acoustic signals induced by the solidifying concrete mixture during the next 10 hours after its mixing. When the concrete mixture is hardened in the open air, the cement stone is additionally hardened as a result of carbonization of calcium hydroxide. The hardened cement stone is a very strong crystalline framework filled with a gel, inside which are the internal layers of cement granules that are not affected by reaction. The pores in the cement stone are filled with air and capillary water. If the cement hydration passes under normal conditions, the resulting calcium hydrosilicates have an alternating composition, that is largely close to amorphous substances and do not form a complete crystalline structure. The resulting material is characterized by good acoustic permeability. Therefore, during the further setting and hardening of cement, that accompanied by insignificant internal volume changes, the AE-signals of shear deformations reach the 
outer surface of the concrete sample. These deformations are detected by a piezosensor in the frequency range of $30-500 \mathrm{kHz}$.

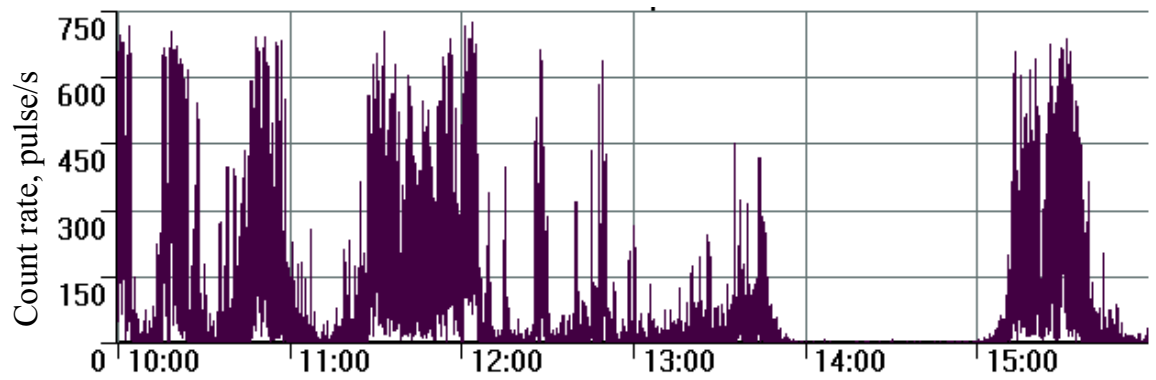

Time, hour

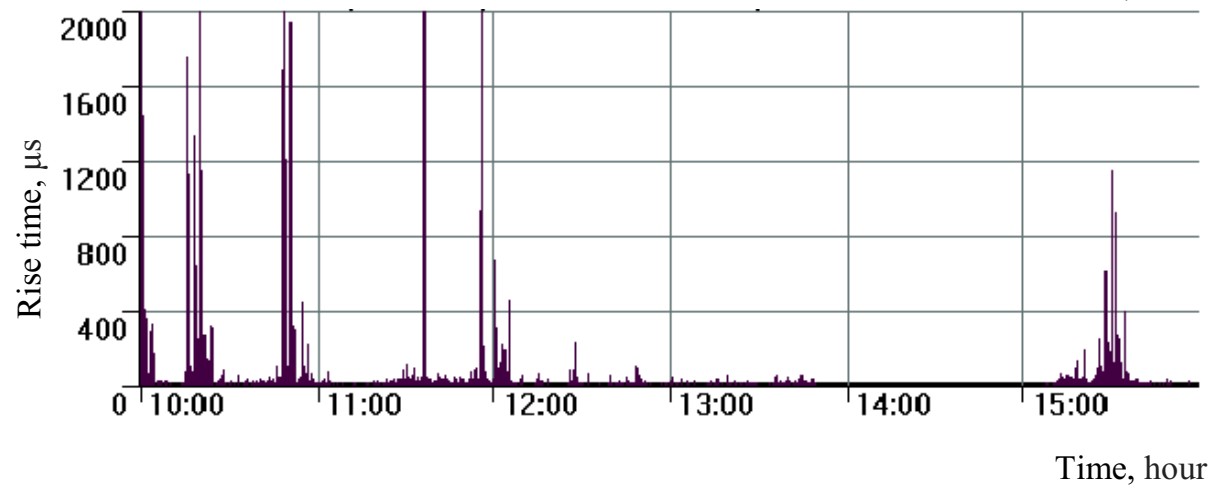

Fig. 5. Change in AE-parameters during setting and hardening of concrete mixture over the time intervals from 10 to 16 hours after its mixing (Source: Author's own figure)

The obtained results can be interpreted as follows. As time pass, the solution becomes supersaturated relative to the hydroferrites. Therefore, the conditions for formation of nuclei of the crystals of cement clinker alumina-ferrite phase and their precipitation from the solution appear in the liquid phase. This causes a decrease of hemihydrate concentration in the liquid phase and creates conditions for the dissolution of new portions of this substance and the formation of a supersaturated solution of the alumina-ferrite phase. The crystals grow, intertwine and grow together, causing setting and hardening of cement mixture. Whereupon there is induction of acoustic signals in the frequency range 100-400 $\mathrm{kHz}$.

\section{Conclusions}

The experimental results show a high informativeness of acoustic method for the concrete mixture condition monitoring. Using of AE-method allows remote monitoring of the dynamics of concrete mixtures condition and the eutectoid transformation of the solid phases. In addition, it has been established that it allows also remote monitoring of the shrinkage processes dynamics in finished product, because each change in the equilibrium condition of thermodynamic system is accompanied by the emission of acoustic signals. Therefore, this method can be successfully applied in building as a non-destructive method for determining the start and end of the solidification of the cement solution. This is especially importance for continuous concrete of civil and industrial buildings and structures, precast structure, road-building, and for the hydraulic structures building. 


\section{References}

1. Guide on the electro-thermal processing of concrete. M. Stroyizdat, Scientific and research institute of concrete and reinforced concrete Gosstroy USSR, (1974)

2. A. Lemarchand, F. Boudoire, E. Boucard, Th. Chotard, A. Smith, J. Phys. Chem., 116, (2012)

3. S.I. Builo, D.M. Kuznetsov, V.L. Gaponov, V.V. Trepachev, Acoustic-Emission Testing and Diagnostics of the Dissolution Kinetics of Crystalline Componet, Rus. J. of Nondestructiv Testing, 48, 10 (2012).

4. S. I. Builo, D. M. Kuznetsov, V.L. Gaponov, Acoustic Emission Testing of Capillary Liquid Flows in Porous Media, Rus. J. of Nondestructiv Testing, 50, 7, (2014).

5. S.I. Builo, D.M. Kuznetsov, V.L. Gaponov, Chapter 13 Acoustic Emission Diagnostics of the Kinetics of Physicochemical Processes in Liquid and Solid Media, In: Advanced Materials. Studies and Applications. Ivan A. Parinov, Shun-Hsyung Chang and Somnik Theerakulpisut (Eds.), New York: Nova Science Publishers, (2015) 